\title{
Quality of Information and Future Directions. Comment on "Influence of Mass and Social Media on Psychobehavioral Responses Among Medical Students During the Downward Trend of COVID-19 in Fujian, China: Cross-Sectional Study"
}

Smriti Sasikumar ${ }^{1}$, BSc; Hafsa Omer Sulaiman ${ }^{1}$, BSc; Simran Bedi ${ }^{2}$; Mikhail Nozdrin ${ }^{3}$; Caroline Rundell ${ }^{4}$; Sadia Zaman $^{3}$

${ }^{1}$ St George's University of London, Cranmer Terrace, London, United Kingdom

${ }^{2}$ King's College London, London, United Kingdom

${ }^{3}$ Imperial College, London, United Kingdom

${ }^{4}$ University College London, London, United Kingdom

Corresponding Author:

Smriti Sasikumar, BSc

St George's University of London

Cranmer Terrace

London

United Kingdom

Phone: 442086729944

Email: Sasikumar.s@ live.sgul.ac.cy

Related Article:

Comment on: https://www.jmir.org/2020/7/e19982/

(J Med Internet Res 2021;23(2):e23168) doi: 10.2196/23168

\section{KEYWORDS}

COVID-19; social media; mass media; information quality

We read with great interest the recent article by Lin et al [1], which investigated the impact of mass and social media on psychobehavioral responses among medical students in China. We thank the authors for investigating this important issue in such a multifaceted manner. As medical students from four different medical schools in London, we questioned whether certain correlations discussed in the study could be examined further to elucidate the results and allow for some extrapolation. The results from this study are quite intriguing, and we believe this deeper analysis could spark other institutions around the world to examine the powerful effect of media on behaviors during a pandemic.

Since the study is based on Chinese media, we were unfamiliar with the quality of disseminated information related to COVID-19, particularly on social media. According to Ren et al [2], while Western media tends to center around controversy and individual experts, Chinese media sources focus on "mainstream positions" put forth by health agencies $[3,4]$. We suggest that an overview of how media sources are presented in China would be extremely useful in contextualizing the results of the study for non-Chinese audiences.
Furthermore, while the article's findings suggest that exposure to mass media and social media could increase positive attitudes and reduce emotional response among university students, we believe that the credibility of the information on these platforms greatly contributes to the results. Misinformation and "fake news," particularly on the internet, have become rampant [5]. The article presumes that the results are due to medical students being more discerning consumers of health information. While this may be possible, we suggest that analyzing the quality of the information consumed by the students, that is, whether the information was credible or not, would allow stronger correlations to be drawn as to why students had positive behavioral responses and lower trait anxiety.

This analysis on the quality of mass and social media could be done in a number of ways. For example, examining the quality of data could involve recruiting some participants from the current study to respond to health information of differing qualities. The information could be put into two categories: credible information and misinformation about COVID-19. Repeating the STAI-6 (Spielberger State-Trait Anxiety Inventory, 6 items) and the psychobehavioral response questionnaire on this controlled set of stimuli might yield more 
data on why mass and social media exposure reduced trait anxiety and changed behavior in the study population.

Lastly, we suggest that the data be further analyzed to examine whether the psychobehavioral and anxiety responses varied by age in the students. This suggestion stems from the observed trend that different age groups engage with different mass or social media platforms. Perhaps the quality and the type of mass or social media may have an effect on the observed results.
Implementing some of these suggestions could use the previously gathered data and provide a uniquely detailed viewpoint that could expand the results of the study. These additions and future steps could inspire cross-institutional collaboration, allowing us to better understand how media across the world can affect the behavior and anxiety levels of key populations during a pandemic.

\section{Editorial Notice}

The corresponding author of "Influence of Mass and Social Media on Psychobehavioral Responses Among Medical Students During the Downward Trend of COVID-19 in Fujian, China: Cross-Sectional Study" did not respond to our invitation to reply to this commentary.

\section{Conflicts of Interest}

None declared.

\section{References}

1. Lin Y, Hu Z, Alias H, Wong LP. Influence of Mass and Social Media on Psychobehavioral Responses Among Medical Students During the Downward Trend of COVID-19 in Fujian, China: Cross-Sectional Study. J Med Internet Res 2020 Jul 20;22(7):e19982 [FREE Full text] [doi: 10.2196/19982] [Medline: 32584779]

2. Ren J, Peters HP, Allgaier J, Lo YY. Similar challenges but different responses: Media coverage of measles vaccination in the UK and China. Public Underst Sci 2014 May;23(4):366-375 [FREE Full text] [doi: 10.1177/0963662512445012] [Medline: 23825271]

3. DeGennaro T. 10 Most Popular Social Media Sites in China 2019. Dragon Social. 2020 Jun 30. URL: https://www. dragonsocial.net/blog/social-media-in-china/ [accessed 2021-02-09]

4. Social Media Fact Sheet 2019. Pew Research Center. 2019 Jun 12. URL: https://www.pewresearch.org/internet/fact-sheet/ social-media/ [accessed 2021-02-09]

5. Sommariva S, Vamos C, Mantzarlis A, Đào L, Martinez Tyson D. Spreading the (Fake) News: Exploring Health Messages on Social Media and the Implications for Health Professionals Using a Case Study. American Journal of Health Education 2018 Jun 07;49(4):246-255. [doi: 10.1080/19325037.2018.1473178]

\section{Abbreviations \\ STAI-6: Spielberger State-Trait Anxiety Inventory, 6 items}

Edited by G Eysenbach, T Derrick; submitted 03.08.20; peer-reviewed by J Li, S Temiz, M K.; accepted 28.01.21; published 18.02.21
Please cite as:
Sasikumar S, Sulaiman HO, Bedi S, Nozdrin M, Rundell C, Zaman S
Quality of Information and Future Directions. Comment on “Influence of Mass and Social Media on Psychobehavioral Responses
Among Medical Students During the Downward Trend of COVID-19 in Fujian, China: Cross-Sectional Study”
J Med Internet Res 2021;23(2):e23168
URL: $\underline{\text { https://www.jmir.org/2021/2/e23168 }}$
doi: $\underline{10.2196 / 23168}$
PMID: $\underline{33599623}$

(C) Smriti Sasikumar, Hafsa Omer Sulaiman, Simran Bedi, Mikhail Nozdrin, Caroline Rundell, Sadia Zaman. Originally published in the Journal of Medical Internet Research (http://www.jmir.org), 18.02.2021. This is an open-access article distributed under the terms of the Creative Commons Attribution License (https://creativecommons.org/licenses/by/4.0/), which permits unrestricted use, distribution, and reproduction in any medium, provided the original work, first published in the Journal of Medical Internet Research, is properly cited. The complete bibliographic information, a link to the original publication on http://www.jmir.org/, as well as this copyright and license information must be included. 